\title{
FINAL HEIGHT OF A GROUP OF PATIENTS WITH CONGENITAL ADRENAL HYPERPLASIA
}

\author{
Marta Almeida Ferreira ${ }^{1}$, Sónia Santos ${ }^{2}$, Ester Pereira ${ }^{3}$, Beatriz Vale ${ }^{4}$, Rita Cardoso ${ }^{4}$, Isabel Dinis ${ }^{4}$, Alice Mirante ${ }^{4}$ \\ ${ }^{1}$ Endocrinology, Diabetes and Metabolism (Centro Hospitalar do Porto), ${ }^{2}$ Paediatrics (Centro Hospitalar de Viseu-Tondela), ${ }^{3}$ Paediatrics \\ (Centro Hospitalar de Leiria-Pombal), ${ }^{4}$ Paediatrics (Hospital Pediátrico de Coimbra - Centro Hospitalar e Universitário de Coimbra)
}

Introduction congenital adrenal hyperplasia (CAH) is a group of autosomal recessive disorders characterized by impaired cortisol synthesis with an incidence of 1:15 000 births. The most common form is attributable to mutations in CYP21A2, the gene encoding the adrenal steroid 21-hydroxilase enzyme (P450c21). Because of that, cortisol synthesis is impaired which leads to a corticotropin stimulation of the adrenal cortex, with accumulation of cortisol precursors that are derived to sex hormone biosynthesis. Aldosterone production may also be decreased (salt wasting forms). The final height of children with these disorders seems to be compromised. That can happen because of the disease itself or because an excessive dose of corticoids used during treatment. This work evaluates the final stature SDS of a group of patients with CAH and correlates it with 17-hydroxyprogesterone levels (170HP) and corticoid dose at 10 years of age and at puberty onset.

Methods selection of children with CAH diagnosed from 1983/08/01 to 1995/08/01, followed in an outpatient clinic of paediatric endocrinology, with all necessary data in their clinical files: height, weight, 170HP and corticoid dose at 10 years of age, puberty onset and at the last medical evaluation, parents height. Data was analysed in SPSS 20.0, with descriptive statistics, Wilcoxon test, Spearman's correlation coefficient and Mann-Witney U test (significance level: 0.05).

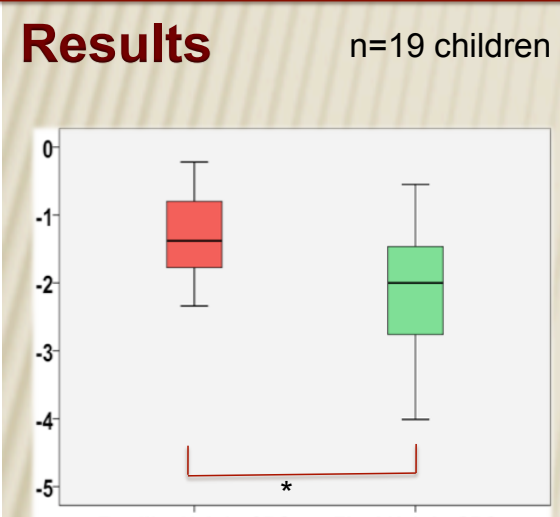

Familiar Height SDS Final Height SDS

$$
\begin{aligned}
& O^{x}=8 \\
& \bigcirc=11
\end{aligned}
$$

13 children were diagnosed in the first year of life 13 children had salt wasting forms of $\mathrm{CAH}$
Graph 1: The mean final height SDS of these children was -2.07 (from -4.01 to -0.55 ; standard deviation, $S D=0.98$ ). Their mean familiar height SDS was -1.33 (from -2.34 to $-0.22 ; S D=0.64$ ). This difference was statistically significant $(p=0.003)$. In centimeters, their mean heigt was 155 (from 141 to $171 \mathrm{~cm} ; \mathrm{SD}=7.44$

Graph 2: At puberty onset, these children's mean -2 height SDS was 0.33 (from -2.6 to 5.14 ; SD=1.76). When they were 10 years old, their mean height -4 SDS was 0.29 (from -1.69 to 3.09; $S D=1.26$ ).

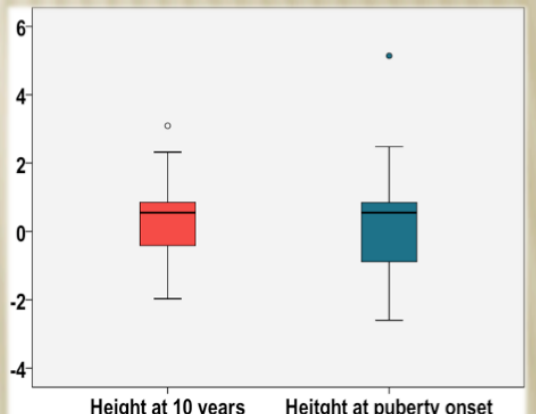

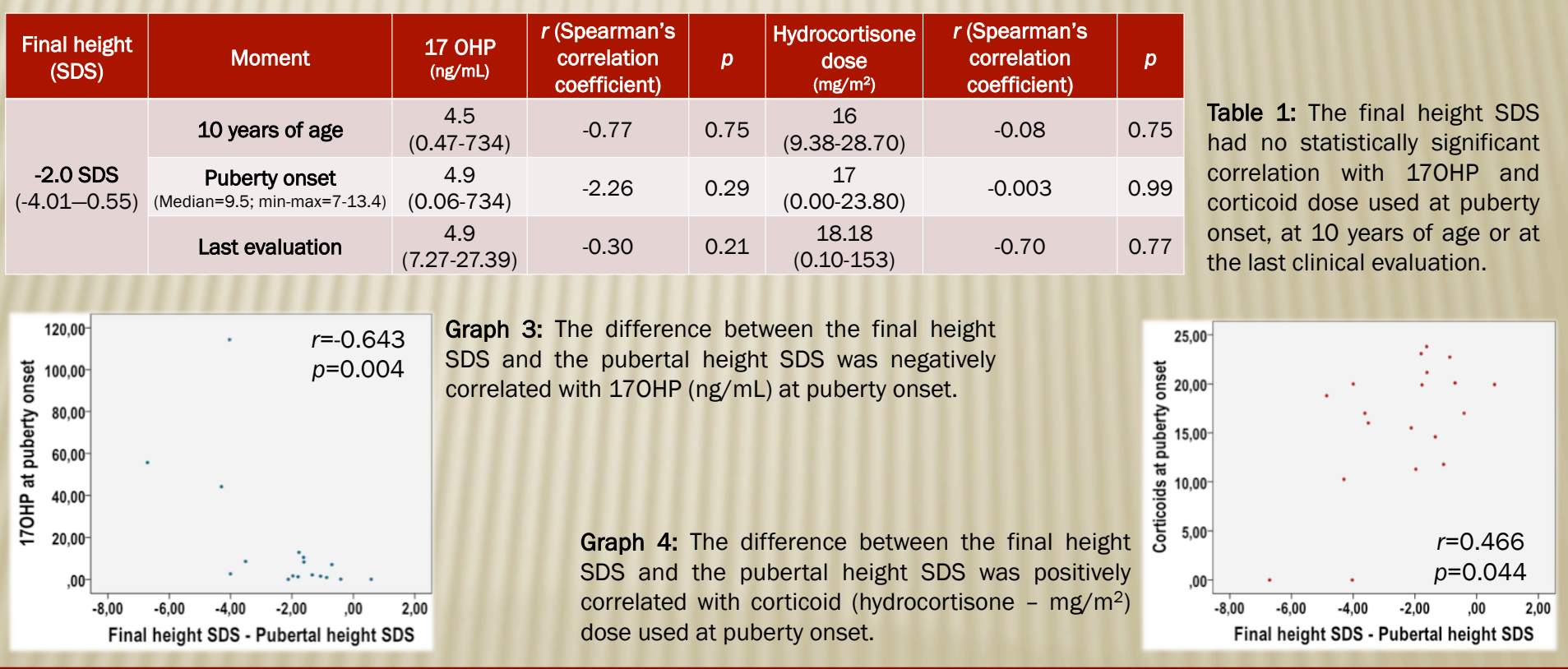

Conclusion

In this group of children the final height SDS was inferior to their target familial stature, because of the poor growth after puberty onset: their height SDS when they were 10 years old and on their puberty onset were better than the final one. There wasn't any correlation with $170 \mathrm{HP}$ and corticoid dose used during treatment. When the growth after puberty onset was analysed, it was positively correlated with corticoid dose and negatively correlated with $17 \mathrm{OHP}$ levels on puberty onset. There are many studies reporting that high doses of corticoids used in these children may impair their growth. But, in this study, to achieve better levels of $170 \mathrm{HP}$, higher doses of corticoids were used. So, maybe that could have helped to achieve a better growth before puberty and could have prejudiced the growth after puberty onset. 